\title{
Network Analyses of the Symptoms in the Coronavirus Anxiety Scale (CAS) and its Associations with Depression, Anxiety, Stress and Alcohol Use
}

\author{
Rapson Gomez \\ Federation University \\ Deon Tullett-Prado ( $\square$ deon.tullettprado@live.vu.edu.au ) \\ Victoria University \\ Shaun Watson \\ Federation University \\ Vasileios Stavropoulos \\ Victoria University
}

\section{Research Article}

Keywords: Coronavirus Anxiety Scale, COVID anxiety symptoms, adults, network analysis, distress, alcohol use.

Posted Date: December 9th, 2021

DOI: https://doi.org/10.21203/rs.3.rs-966118/v1

License: (9) This work is licensed under a Creative Commons Attribution 4.0 International License.

Read Full License 


\section{Abstract \\ Background}

The Covid-19 Pandemic and subsequent actions taken by national/international organizations has generated a large amount of anxiety which may roam into the realm of pathology - COVID Anxiety. In order to measure this phenomenon, measures such as the CAS have been developed. The CAS being a self-report measure of anxiety-related physiologically symptoms that are aroused by information and thoughts related to COVID-19. However, as the CAS is fairly new tit requires validation and examination. This study fulfils this need through the use of Network Analysis.

\section{Methods}

The study used regularized partial correlation network analysis (EBICglasso) to examine the network structure of ratings of COVID anxiety symptoms as presented in the Coronavirus Anxiety Scale (CAS) and how these symptoms are related to distress (combination of depression, anxiety, stress) and alcohol use. A total of 968 adults from an Australian community sample completed the CAS, and measures of depression, anxiety, stress and alcohol use.

\section{Results}

The findings showed that the most central CAS symptom was abdominal distress, followed by tonic immobility. The symptom with the lowest strength centrality value was dizziness. Also, the network revealed at least moderate effect size connections for tonic immobility with dizziness, sleep disturbances abdominal distress, and for abdominal distress with appetite loss. Additionally, distress was associated positively with dizziness, tonic immobility and appetitive loss. Alcohol use was associated positively with dizziness and abdominal distress, and negatively with tonic immobility and appetitive loss.

\section{Conclusions}

Overall, the findings showed a novel understanding of the structure of the COVID anxiety symptoms in the CAS, and how these symptoms are associated with distress and alcohol use. The clinical implications of the findings for assessment and treatment of COVID anxiety and its comorbidity with distress and alcohol use are discussed.

\section{Background}

COVID-19 and its related pandemic is having a serious impact on the mental health of many individuals (Huang \& Zhao, 2020; Pfefferbaum \& North, 2020). A systematic review of 19 studies concluded associations with anxiety, stress, posttraumatic stress disorder, depression, and psychological distress 
(Xiong J, Lipsitz O, Nasri F, et al., 2020; see also the review by Salari et al., 2020). This has also been noticed in Australia (Dawel et al, 2020), where the current study was conducted. In addition to these traditionally recognized psychopathologies, the pandemic has caused many people to develop anxiety and fear of being infected with the COVID virus (McKay, Yang, Elhai, Asmundson, 2020), leading them to respond negatively to coronavirus related thoughts or information (Lee, Mathis, Jobe, \& Pappalardo, 2020). This reaction has been referred to as COVID anxiety or "coronaphobia" (Arora et al., 2020). Related to this, Lee (2020) developed the Covid Anxiety Scale (CAS) in order to measure anxiety-related physiological (somatic) symptoms that are aroused by information and thoughts related to COVID-19. This measure has been validated multiple times and is now considered a valid measure for screening COVID 19 related anxiety (Nikčević \& Spada, 2020). Indeed, our examination of Google Scholar indicated a total of 614 citations as of 21 September 2021, however the measure is still relatively new and thus requires examination. In the current study, we used a novel technique called network analysis (Epskamp, Borsboom, \& Fried, 2018) to examine the structure of the CAS anxiety symptoms, and how these symptoms are related uniquely with distress (combination of depression, anxiety, stress) and alcohol use.

\section{COVID Anxiety: Description, Impact and Measurement}

Arora et al. (2020) has noted that while coronaphobia is like other phobias (i.e., characterized by fear of a specific stimuli, dysfunctional and distorted thoughts, and avoidance responses), it is unique in that the fear is not only limited to public places/situations/objects, but also appears when coming in physical contact with humans. According to Arora et al. (2020), COVID anxiety has three primary interacting components: physiological (i.e., triggering of the fight or flight response when exposed to antecedent event, accompanied by worry that palpitations, tremors, difficulty in breathing, dizziness, change in appetite, and sleep ); cognitive (fear of virus casing preoccupation with threat provoking cognitions); and behavioral (preventive responses such as avoidance).

Evidence indicates that individuals prone to higher levels of COVID anxiety have a higher risk of developing severe mental health problems (Arora et al., 2020), including depression, stress, anxiety (Xiong et al., 2020; see also the review by Salari et al., 2020), and alcohol use (Gasteiger et al., 2021; Stanton et al., 2020), and that these may last well beyond the course of the pandemic (Nikčevića \& Spada, 2020).

To date, several other measures have also been developed for measuring COVID-19 anxiety (and fear). They include the Fear of COVID-19 Scale (FCV-19S; Ahorsu et al., 2020), the COVID Stress Scales (CSS; Taylor et al., 2020), Fear of the Coronavirus Questionnaire (FCQ; Mertens et al., 2020), and the Covid Anxiety Scale (CAS; Lee, 2020a). A recent study showed that the FCV-19S, the FCQ and the FCQ scales/subscales measure different aspects of COVID anxiety and fear, thereby indicating that COVID anxiety is heterogeneous. Given these findings, it can be argued that for a clear understanding of COVID anxiety, researchers need to identify what aspect of COVID anxiety their study is focusing on, and then consider this when interpreting their findings (Mertens et al., 2020).

\section{CAS}


The CAS is a 5-item self-report measure of anxiety-related physiologically (somatic) symptoms that are aroused by information and thoughts related to COVID-19. The five items of the CAS, all loading on a single factor, are (1) I felt dizzy, lightheaded, or faint, when I read or listened to news about the coronavirus (dizziness); (2) I had trouble falling or staying asleep because I was thinking about the coronavirus (sleep disturbances); (3) I felt paralyzed or frozen when I thought about or was exposed to information about the coronavirus (tonic immobility); (4) I lost interest in eating when I thought about or was exposed to information about the coronavirus (appetite loss); and (5) I felt nauseous or had stomach problems when I thought about or was exposed to information about the coronavirus (abdominal distress). According to Lee (2020b), the dizziness and tonic immobility symptoms in the CAS capture the physiological reactions of elevated fear to corona virus related stimuli. The sleep disturbances and appetite loss symptoms capture the physical effects of excessive worry about the coronavirus. The abdominal distress symptom captures fear and anxiety, result from a fearful reaction or the physical effect of excessive worry, or both.

Upon its construction, number of validation studies have examined the CAS, finding good psychometric properties. The initial CAS development and validation study supported a unidimensional structure, with high reliability $(a=.93)$. Scores on the CAS correlated in theoretically meaningful ways with coronavirus diagnosis, functional impairment, coping through substance use and religion, hopelessness, and suicidal ideation. ROC analysis indicated $90 \%$ sensitivity and $85 \%$ specificity for detection and classification with a cutoff point $\geq 9$ (Lee, 2020a). Based on a subsequent study (Lee, 2020b), the cutoff score was reduced to $\geq 5$. To date numerous other studies have provided additional support for the psychometric properties of the CAS (e.g., Evren et al., 2020; Lee et al., 2020; Skalski et al., 2020). For example, Lee et al. (2020) found that dysfunctional scores on the CAS were associated with coronavirus infection, generalized anxiety, depression, functional impairment, perceived lack of social support, and suicidal ideation. For a full list of research studies that have used the CAS, the reader is referred to https://sites.google.com/cnu.edu/coronavirusanxietyproject/home.

Overall, therefore the CAS has sound psychometric properties. Although COVID anxiety is a heterogeneous construct, the CAS with its five somatic items, is essentially a unidimensional, tapping physiologically and somatic anxiety and fear. As ready noted, despite it brevity and undimensionality the CAS is considered a valid measure for screening COVID 19 anxiety and has been used widely in COVID anxiety research. Therefore it can be argued that the CAS is ideally suited for examining the somaticrelated anxiety symptoms of COVID anxiety. Focusing on this group of symptoms is important as there is now some evidence that moderate to high levels of COVID-19 anxiety is associated with more somatic symptoms, even after controlling for generalized anxiety disorder (GAD), preexisting health problems, age, gender, and income.

\section{A Novel Approach for Examining the Psychometric properties of the CAS}

To date, the psychometric properties of CAS have largely been examined from a latent variable perspective. In this perspective, it is assumed that there is a latent (unobservable) construct (which is the 
disorder/problem in question) that causes a range of observable responses (that are the symptoms of the latent disorder/problem). This is a reflective view of psychopathology. Seen in the context of COVID anxiety, the reflective view suggests that the COVID anxiety symptoms are responses arising from an assumed underlying latent COVID anxiety construct. This means that the COVID anxiety symptoms are interchangeable and equally reflective of latent COVID anxiety. Also, the COVID anxiety symptoms are considered to have nothing in common after controlling for the latent construct (an assumption referred to as local independence).

Although the latent variable approach (like that captured in a CFA) is currently the most dominant approach for understanding psychopathologies and related syndromes, a newly developed perspective, called the network approach, has a different view of psychopathologies. In the network framework, symptoms are understood as a causal system, interacting with each other in meaningful ways, resulting in the disorder or the syndrome (Borsboom \& Cramer, 2013). A network model can be tested empirically using 'network analysis' (Borsboom \& Cramer, 2013; Boschloo et al., 2015). Network analysis is an exploratory approach that provides visual and quantitative information about symptoms that are "core" or "central" (important) to the overall network of symptoms, and the strength of connections between symptoms (Borsboom \& Cramer, 2013; Fried et al., 2015). As noted by Epskamp and others (Epskamp \& Fried, 2018; Epskamp et al., 2017), such a network can identify unique interactions between variables that cannot be identified using multiple regression analysis, and when the network analysis is exploratory it is advantageous over structural equation modelling (SEM), because there are no equivalent undirected models possible in SEM.

\section{Clinical Importance of Network Analysis of the COVID Anxiety Symptoms}

Results from network analysis of the symptoms of a disorder/syndrome can have important implications for theory, assessment and diagnosis, treatment and prevention. Traditionally, the theoretical importance of a symptom is viewed in terms of its severity which is ascertained in terms of its mean score. However, in network models, centrality, that is different from mean score, defines the importance of a symptom. Indeed, the mean levels of symptoms can change without changes in their centrality in the network (Yang et al., 2016). Thus, different conclusions about what are core symptoms in a disorder/syndrome could be arrived at when looking at symptom centrality and symptom severity (Mullarkey et al., 2019).

In relation to treatment, as symptoms for a disorder/syndrome identified as central in a network are considered most influential in producing or maintaining the disorder/syndrome, intervening on these symptoms can be expected to maximize the impact of intervention. In this respect, and given its network characteristic, focusing on the central symptoms could potentially have a downstream effect in improving other network symptoms. Specific to COVID anxiety, as note by Ramos-Vera (2021), network analysis allows clinicians to identify and understand more accurately the most important components in the dysfunctional dynamics of COVID anxiety, and consequently, findings from network analysis, can contribute more effectively to detection and intervention of the negative effects of COVID-19. 
Also, related to treatment, an expended network model that includes the COVID anxiety symptoms with other psychopathologies will increasing our understanding of the development and maintenance of other comorbid psychopathologies, which in turn would have major implications for preventing and treating these comorbidities.

\section{Existing Network-based Psychometric Data for the CAS and for COVID Anxiety in General}

To date, as far as we were able to establish, there have been only one study that has examined the network structure of COVID anxiety symptoms in the CAS (Ramos-Vera, 2021). The network was examined in terms of regularized partial correlation coefficients for ratings provided by a Peruvian community sample. The results indicate that appetite loss (symptom \#4) was most central (indicating that it has greatest influence in the network) and featured a strong connection (called edge weight) with dizziness (item \# 1). Additionally, sleep disturbances (item \#2) and tonic immobility (item \# 3); and appetite loss (item \# 4), and abdominal distress (item \# 5) were also strongly connected with each other indicating a stronger influence upon each other than they would have with less strongly connected symptoms.

Two other studies have used network analysis to examine COVID-19 related anxiety and fear (Mertens et al., 2021; Taylor et al., 2020). Mertens et al. (2021) applied network analysis concurrently to the scales/subscales of the FCV-19S, CSS, FCQ, and a measure related to COVID-19 worries. They reported four clusters: fear of health-related consequences, fear of supplies shortages and xenophobia, fear about socio-economic consequences, and symptoms of fear (e.g., compulsions, nightmares). The most central cluster was that related to fear of health. Taylor et al. (2020) applied network analysis on CSS variables together with other variables related to COVID-19 (e.g., avoidance, self-protective behaviors, stockpiling and panic buying; use of personal protective equipment, and belief in COVID-19-related conspiracy theories). The CSS variables were (1) worry about the dangerousness of COVID-19 (2) worry about the socioeconomic consequences of COVID-19 (3) xenophobic fears that foreigners are spreading Covoid-19, (4) traumatic stress symptoms associated with direct or vicarious traumatic exposure to COVID-19 and (5) COVID-19-related compulsive checking and reassurance-seeking. They reported three major clusters, with a cluster related toworries about the dangerousness of COVID-19 being most central, followed by the cluster related to the belief that the COVID-19 threat is exaggerated. The third cluster was related to compulsive checking, reassurance-seeking, and self-protective behaviors. Taken together, while these studies proved valuable information on the heterogeneous nature of anxiety and fear related to COVID-19, they offer no specific network information on the CAS.

\section{Limitations of Existing Network-Based Data on the CAS}

Although there is some network analysis data on the COVID anxiety symptoms, as presented in the CAS, the findings are limited. Firstly, there is only one study (Ramos-Vera, 2021). As network analysis is based on classical test theory, the findings from such studies are largely sample dependent. Thus is need for replication studies. Secondly the only existing study that has used network analysis to examine the CAS (Ramos-Vera,2021), did not examine and report the accuracy and stability of the findings for centrality 
and edge weights (connections between symptoms). This is a limitation as network analysis experts have recommended that a network must also be evaluated for its accuracy and stability (Epskamp et al., 2018). Thirdly, the Ramos-Vera (2017) study did not examine or illustrate how the network model would be used to examine how COVID anxiety contributes to the development and maintenance of other comorbid psychopathologies, which in turn would have major implications for understanding, preventing, and treating these comorbidities. For example, as already noted, pandemic-related psychological anxiety and distress are related to elevated levels of depression, stress, anxiety or distress (Xiong et al., 2020; see also the review by Salari et al., 2020), and alcohol use (Gasteiger et al., 2021; Stanton et al., 2020). From a network perspective, the inclusion of such distress and alcohol use together with the CAS symptoms in the same network model will reveal the specific CAS symptom or symptoms that are central to the development and maintenance of these comorbidities, and therefore identify important targets of intervention. Fourthly, to date there is no network data for COVID anxiety symptoms in a Western community. Given these limitations, there is clearly need for more network analysis studies using Western samples and examining network findings for accuracy and stability, and also relationships for CAS symptoms with potential comorbidities.

\section{Aims of the Present Study}

Given the limitations in existing network data on the CAS, and the positive clinical contributions that network analysis can offer, the major aim in the current study was to use network analysis, with regularized partial correlation, to examine the network structure of the five COVID anxiety symptoms in the CAS (dizziness, sleep disturbances, tonic immobility, appetite loss, and abdominal distress) in a large Western (Australian) community sample. In the current study, we produced a network graph, displaying the topology of the symptom network, comprising the five CAS somatic symptoms. We then evaluated statistically (using both edge width and centrality) the respective influence of the symptoms in the network; and the robustness and stability of the network findings. A secondary aim of the study was to compute an expended network model that included the COVID anxiety symptoms with distress and alcohol usage to ascertain the major associations of the COVID anxiety symptom with distress and alcohol usage.

\section{Method}

\section{Participants}

Participants were 968 English speaking Australian adults from an online convenient sample from the general community. The age of participants ranged from 18 to 64 years (mean $=29.54$ years; $S D=9.35$ years), and included 622 men (64.3.7\%; mean age $=29.46$ years, $S D=8.93$ years), and 315 women (32.5\%; mean age $=30.02$ years, $S D=10.39$ years). Additionally, 26 individuals $(2.7 \%)$ identified themselves as trans/non-binary gender, 1 individual identified as queer, and 4 individuals did not specify their gender. No significant age differences were found across men and women, $t(935)=0.846, p=.398$. In terms of sociodemographic background, slightly more than half the number of participants reported 
being employed (55.0\%) and most of them reported having completed at least secondary education (98.2\%). Based on recommended scores of scores of $\geq 9$ (Lee, 2020a) and $\geq 5$ (Lee, 2020b), the number of individual screening positive for COVID anxiety were $35(3.62 \%)$ and $123(12.70 \%)$, respectively.

\section{Measure}

Coronavirus Anxiety Scale (CAS; Lee, 2020a)

The CAS was used to measure COVID anxiety. It is a 5-item self-report measure of anxiety-related physiologically symptoms that are aroused by information and thoughts related to COVID-19.

Participants rate each item in terms of how frequently they experience each anxiety symptom over the previous two weeks on a 5-point scale, ranging from 0 (Not at all) to 4 (Nearly every day over the last 2 weeks). Thus, higher scores indicate higher levels of a COVID-19 anxiety. The CAS has shown good reliability and validity (Lee et al., 2020). Although the Cronbach a for this scale in the current study was relatively low at .683 , the McDonald omega coefficient was high at .87 .

\section{Depression Anxiety Stress Scales-21 (DASS-21; Lovibond \& Lovibond, 1995)}

The 21-item DASS-21 is a self-report measure with sub-scales for depression, anxiety, and stress. The anxiety items make no reference to COVID anxiety. All 21 items are rated on a 4-point scale $(0=$ did not apply to $3=$ applied most of the time) in terms of how often the individual experienced the behavior during the past week. Although past evidence has shown acceptable convergent and discriminant validities, and high internal reliabilities for the DASS-21 sub-scales (Lovibond \& Lovibond, 1995; Norton, 2007), more recent studies have supported a bifactor model, with a dominant general factor on which all the depression, anxiety and stress items load (e.g., Gomez et al., 2013). Thus, the DASS-21 items can be considered to measure general distress (Lee et al., 2019). The combined anxiety, depression and stress scale scores was used in the study to measure distress. The Cronbach's alpha for the full DASS-21 measure was 0.95 in the current study.

\section{Alcohol Use Disorders Identification Test (AUDIT; Babor et al., 1992)}

Developed by the World Health Organization (WHO), the AUDIT is a 10-item self-report questionnaire with questions regarding amount and frequency of drinking, symptoms of alcohol dependence, and alcoholrelated problems. Each item is rated on a 4-point scale, ranging from 0 (never) to 4 (daily or almost daily). Thus, higher scores indicate more severity. The total score for the 10 items is generally used as an overall measure of the severity of hazardous or problematic drinking, and was used in the current study to measure alcohol addiction behavior. The AUDIT has good reliability and validity (Saunders et al., 1993). The internal reliability (Cronbach's alpha) for the total score for the current study was 0.89 .

\section{Procedure}

This study was approved by the Human Ethics Research Committee, Victoria University (Australia). In order to gather participants, the study was advertised widely with Qualitrics links provided for participants 
to register their interest via social media (i.e. Facebook; Instagram; Twitter), the Victoria University websites and digital forums (i.e., reddit.com). The link took them to the Plain Language Information Statement (PLIS). Those wishing to participate were directed to click a button to agree to informed consent. This was followed by the questions seeking sociodemographic information, and the study questionnaires. Participants completed the online survey using a computer in a location of their choosing.

\section{Statistical Network Analyses}

In network analysis, variables are referred to as nodes, and the relationships between the nodes are referred to as edges. The strength of the relationship between nodes is indicated in terms of edge weights. Network nodes and edges can be estimated using zero-order correlations. In such instances, the edges between nodes will not control for the relations with other nodes, thus inflating correlations, and therefore resulting in difficult to interpret and misleading results. To overcome this, a regularized partial correlation approach, such as the graphical Least Absolute Shrinkage and Selection Operator (g-lasso; Tibshirani, 1996) is used to compute network analysis. Lasso shrinks small partial correlations to 0 , resulting in a sparse network, and showing only the most important relationships in it. When a lassobased approach is applied, there is generally low likelihood of false positives, thereby providing confidence of edges reported in the network (Krämer et al., 2009). However, lasso can result in false negatives, and therefore the absence of an edge between two nodes cannot be automatically assumed to mean that there is no relation between them.

For the current study, we used the network module provided in Jeffreys' Amazing Statistics Program (JASP) version 0.14.1.0 statistical software (Team, JASP, 2018) to conduct the network analysis. JASP uses the bootnet (Epskamp et al., 2018) and the qgraph (Epskamp et al., 2012) packages from $\mathrm{R}$ to conduct network analyses and network graphs, respectively. We applied the extended bayesian information criterion (EBIC) glasso for the network analysis, as used in other studies (Heeren et al., 2018; Isvoranu et al., 2017; McNally et al., 2017). The EBICglasso produces the optimal degree of shrinkage according to an EBIC and a hyperparameter. The hyperparameter in the study was set at 0.5 , since it is suggested to produce networks that balance specificity and interpretability with sensitivity (Foygel \& Drton, 2010; Epskamp \& Fried, 2018). Consequently, the network produced a model that is sparser and easier to interpret, blue edges were indicative of positive relations, and red edges were indicative of negative relations. Additionally, edge weights (strength of the relations between nodes) are shown in terms of varying the thickness and color density of the edge connecting the nodes, with thicker denser colored lines indicating stronger relationships. The distance between nodes is indicative of the relationship between them (i.e., nodes with stronger similarities are close together). Also, as the qgraph package applies the Fruchterman and Reingold's (1991) algorithm to position the nodes, nodes with stronger correlations are positioned near the center of the network, while nodes with weaker correlations are positioned near the periphery of the network. Additionally, nodes with stronger intercorrelations are positioned closer to one another, while nodes with weaker connections are spaced further apart. 
Apart from visualization of the network graph, the network can be described statistically in terms of edge weights and centrality of the nodes (Borgatti, 2005). An edge weight indicates the strength of the relationship between nodes in terms of partial correlations coefficients. Centrality refers to the relative importance of the individual nodes in the network, i.e., a symptom with high centrality is one that is highly connected to other symptoms, and it may be the case that a central symptom is being activated by other symptoms. In contrast, a symptom with low centrality has fewer connections with other symptoms, and has less influence on the network. Thus, while the network graph displays the topology of the symptom network, centrality indicates the relative importance of individual nodes within the network. Edge weights and the position of a node in the network determine its centrality.

Three commonly reported indices of centrality are betweenness, closeness, and degree (called strength in a weighted network, as is the case in the current study), (Opsahl et al. 2010). For reason that we will explain later, we used strength as our measure of centrality. In brief, strength is the sum of all direct associations a given symptom exhibits with all other nodes; and it reflects the direct influence a given node has on the network. Nodes with high strength centrality values indicate that they are more central. Strength is known to reflect reasonably precise centrality estimates for psychology networks (Santos et al., 2018).

A network must also be evaluated for its accuracy and stability. A network's accuracy and stability refer to the likelihood that the network results will be replicated. For this, it has been recommended that the accuracy of the edge and the stability of the centrality estimates should be examined. One way to estimate the accuracy of edge weights is using bootstrap 95\% non-parametric confidence intervals (Cls) (Epskamp et al., 2018). Narrower Cls suggest a more precise estimation of the edge (Epskamp et al., 2018). The stability of the centrality indices can be examined by using a different type of bootstrapping referred to as case-dropping (or alternatively node-dropping) bootstrapping (Epskamp et al. 2018). This procedure examines if the order of centrality indices remains the same after re-estimating the network with less cases (or nodes). It quantifies the stability of centrality indices in terms of correlation stability coefficient. This coefficient reflects the correlation between the original centrality indices (based on the full data) and the correlation obtained from the subset of data representing different percentages of the overall sample. Although a correlation stability coefficient of 0.7 or higher has been suggested as being the threshold, Epskamp et al. (2018) have suggested that the correlation stability coefficient should not be below 0.25 , and preferably it should be above 0.5 . For the current study, the stability of the centrality indices and edge accuracy of the network were examined using the procedures just described. Both were estimated with 1000 bootstrap samples.

\section{Results}

\section{Descriptive information of Data}

There was no missing data. Initially we examined the mean and standard deviation $(S D)$ scores. The findings are presented in Table 1. As show, the mean score for the five symptoms ranged from 0.23 to 
0.48 . The two most severely rated symptoms were sleep disturbances (2) and abdominal distress (5), and the symptom with the lowest severity was dizziness (1). Inspection of the distributions of the frequencies of the categories for each symptom indicated all five response options for all five symptoms were endorsed. Thus, it can be assumed that the ratings provided captured the full trait spectrum of the CAS symptoms.

\section{Visualization of the COVID Anxiety Network}

Figure 1 shows a visualization of the network of the five CAS symptoms. As shown, all symptoms were associated positively (blue edges) with one another, thereby indicating that all the symptoms were associated positively with each other.

\section{Edge Weight of COVAD anxiety Symptoms in the COVAD Anxiety Network}

Table 1 shows that weights matrix between the CAS nodes from the network analysis. For ease of interpretations, based on Ferguson's (2009) effect size guidelines for correlation $(r)$ values $(\leq 0.2$, small; > $0.2<0.5$, moderate; $\geq 0.5$, large), we interpreted $r \geq 0.3$ (moderate effect size) as important. As shown in this table, although all connections were significant, the edge weights meeting the moderate effect size cut-off were for tonic immobility (3) with dizziness (1), sleep disturbances (2) and abdominal distress (5). The connection between abdominal distress (5) with appetite loss (4) of large effect size. The accuracy of the edge weights, estimated using bootstrap 95\% non-parametric Cls is shown in Supplementary Figure S1. As shown, the $\mathrm{Cl}$ ranges around all but four of the estimated edge-weights did not include zero thereby indicating fairly good precision for the edge weights. The $\mathrm{Cl}$ of the four edges that included zero were dizziness-appetite loss, tonic immobility-appetite loss, dizziness-sleep disturbance, and dizziness-abdominal distress. Thus, caution is needed when interpreting these edges in the network.

\section{Centrality of the Symptoms in the CAS Network}

Prior to examining the centrality of the symptoms, we examined stability of the centrality indices for betweenness, closeness, and strength using case-dropping bootstrapping. The findings are displayed in Supplementary Figure S2. The figure shows that for all centrality indices, the correlation stability (CS) coefficient from the subset of data representing different percentages of the overall sample.

Supplementary Figure S2 shows that there was a slight drop in the correlations between the subsample estimate and the estimate from the original entire sample as the subset samples decreased from $95 \%$ of the original sample to $25 \%$ of the sample. However, for this, the correlations for the centrality indices for strength remained above .7 for decrease from $95 \%$ of to $25 \%$ of the sample, thereby indicating stability for the strength centrality indices (Epskamp et al., 2018). Given, this we examined centrality of the symptoms using only strength.

The standardized estimates of the centrality indices for strength are presented in Table 2. To ease interpretation, plots for the centrality measures in terms of $z$ scores were created, and this is displayed in 
Fig. 2. For those interested, both Table 2 and Figure 2 also present the centrality indices for betweenness and closeness. As shown in Fig. 2 and Table 2, the two symptoms (in descending sequence) with the highest strength centrality values were abdominal distress (5) and tonic immobility (3). The symptom with the lowest strength centrality value was dizziness (1). These were also the case when the expected influence centrality values were considered.

\section{Network Analysis for the Associations of the CAS Symptoms with Distress and Alcohol Usage}

In the network analysis model that examined the associations of the CAS Symptoms with distress and alcohol usage, the five CAS symptoms, the DASS (Lovibond \& Lovibond, 1995) distress scores, and the total sore for the AUDIT (Babor et al., 1992) were subjected simultaneously to network analysis. Table 3 shows the weights matrix from this analysis. As shown in this table, distress was associated positively with dizziness (1), tonic immobility (3) and appetitive loss (4). Alcohol use was associated positively with dizziness (1) and abdominal distress (5), and negatively with tonic immobility (3) and appetitive loss (4).

\section{Discussion}

The current study used network analysis to examine the structure of the five COVID anxiety symptoms as measured by the CAS in a group of adults from the general Australian community. It examined the centrality of each COVID anxiety symptom in the network; the edge weights for the COVID anxiety symptom pairs, and the stability and accuracy of indices for centrality and edges. Additionally, the study examined how the COVID anxiety symptoms in the CAS were related to distress (combined depression, anxiety, and stress) and alcohol use.

\section{COVID Anxiety Network Findings}

Initially we examined the stability of the centrality indices (Epskamp et al., 2018), and in terms of strength discovered the following. The highest strength centrality values were abdominal distress, followed by tonic immobility. The symptom with the lowest strength centrality value was dizziness. It therefore follows that abdominal distress and tonic immobility may be the more important COVID anxiety symptoms, and dizziness may be the least important COVID anxiety symptoms. As there was support for the reliability for the strength centrality, these findings can be considered as reliable.

The edge weight findings showed that all connections were significant, thereby indicating associations with each other. Furthermore, the edge weights meeting at least the moderate effect size cut-off were for tonic immobility with dizziness, sleep disturbances and abdominal distress, and abdominal distress with appetite loss. As the test for the accuracy of the edge weights estimated using bootstrap 95\% nonparametric $\mathrm{Cl}$ did not include zero for these edges, there findings can be considered as reliable.

\section{Extended (CAS Symptoms with Anxiety, Depression, Stress, and Alcohol Usage)}

In the network analysis model that examined the associations of the CAS Symptoms with distress and alcohol usage, the five CAS symptoms, the DASS (Lovibond \& Lovibond, 1995) distress scores, and the 
total sore for the AUDIT (Babor et al., 1992) were subjected simultaneously to network analysis. Our findings showed that distress was associated positively with dizziness, tonic immobility and appetitive loss. Alcohol use was associated positively with dizziness and abdominal distress, and negatively with tonic immobility and appetitive loss.

\section{Comparison of Current and Past COVID Anxiety Network Findings}

The findings in the current study differ from the only previous study in this area by Ramos-Vera (2021). Although that study also examined the network in terms of regularized partial correlation coefficients, it found, unlike the current study, that appetite loss was most central, and there were strong connection for appetite loss with dizziness, sleep disturbances and tonic immobility; and appetite loss and abdominal distress. Existing data show cultural and cross-national differences in the level of anxiety reported during the pandemic (Burkova et al., 2021; Chen et al., 2021; Shah et al., 2021). Given this, it is possible that such differences could explain (at least in part) the differences between our findings and that reported by Ramos-Vera (2021). As mentioned previously, Ramos-Vera (2021) examined a Peruvian sample. In contrast, the current study examined an Australian sample. If so, it would imply that cultural factors may be important contributors to COVID anxiety network findings and therefore COVID anxiety. At a more general level, this would mean that cultural factors need to be considered when treating COVID anxiety.

Another major difference is that the current study we examined how the COVID anxiety symptoms in the CAS were related to distress and alcohol use. Ramos-Vera (2021) did not extend their exploration in a similar manner. Thus, the current study provided a more a useful and valuable understanding of how the symptoms in the CAS-network influence the development of comorbid distress and alcohol use (Gasteiger et al., 2021; Stanton et al., 2020; Xiong et al., 2020).

\section{Novel Clinical Implications}

Our findings have novel implications for theory, classification, assessment and diagnosis, and treatment and prevention. We focus here on the major implications.

First, in a network, symptoms with high centrality values are considered most influential in producing or maintaining the disorder/ syndrome. The highest strength centrality values were abdominal distress, followed by tonic immobility. Thus, it can be argued that the abdominal distress and tonic immobility symptoms are especially important for understanding and managing COVID anxiety, and therefore individuals with serious problems related to abdominal distress and tonic immobility are likely to demonstrate more serious COVID anxiety presentations. Therefore, clinicians may wish to pay special attention to the presence of these symptoms during assessment and diagnosis of COVID anxiety. As the symptom with the lowest strength centrality value was dizziness, it can be speculated that this symptom may not be critical for COVID anxiety.

Second, traditionally, the theoretical importance of a symptom is viewed in terms of its severity which is ascertained in terms of its mean score. The two most severely rated symptoms were sleep disturbances 
and abdominal distress. Given that in the network analysis, the highest two centrality symptoms were abdominal distress and tonic immobility, somewhat different conclusions about what are core symptoms in COVID anxiety were found when looking at symptom centrality and symptom severity (Mullarkey et al., 2019). Thus, it will be useful for clinicians to also consider symptom centrality when assessing and treating individuals with COVID anxiety.

Third, because the symptoms with high centrality values are considered most influential, intervening on these symptoms can be expected to influence other symptoms and in that way reduce the impact of the other symptoms also. This, therefore, could mean that focusing intervention efforts on abdominal distress and tonic immobility symptoms rather than the other symptoms could facilitate treatment effects. Where relevant, focusing on the symptoms with high centrality values (abdominal distress and tonic immobility) may also reduce the chances of on-set and development of COVAD anxiety in the context of primary prevention protocols implemented in the community.

Fourth, the edge weight findings suggest that tonic immobility is more likely than the other symptoms to be associated with dizziness, sleep disturbances and abdominal distress, and also that abdominal distress is more likely than the other symptoms to be associated with appetite loss was of large. It would be useful for clinician to keep these associations in mind when assessing and treating individuals referred for COVID anxiety. Seen together with the findings for centrality, it can be speculated that abdominal distress (feeling nauseous or having stomach problems when thinking about or being exposed to information about the coronavirus) and tonic immobility (feeling paralyzed or frozen when thinking about or being to information about the coronavirus) are probably the symptoms most influential in COVID anxiety. According to Lee (2020b), the tonic immobility symptoms in the CAS capture the physiological reactions of elevated fear to corona virus related stimuli; and the abdominal distress symptom captures fear and anxiety, result from a fearful reaction or the physical effect of excessive worry, or both.

Fifth, the extended network model showed that the dizziness symptom was associated positively with distress and alcohol use; the abdominal distress symptom was associated positively with alcohol use; and the tonic immobility and appetite loss symptoms were associated positively with distress. This indicates that higher levels of the dizziness symptom will be associated with distress and alcohol use, higher levels the abdominal distress symptom will be associated with alcohol use; and higher levels of tonic immobility and appetite loss symptoms will be associated more distress. It would be useful for clinician to keep these associations in mind when assessing and treating individuals referred for COVID anxiety.

\section{Limitations and Directions for Further Studies}

Despite the positive value of the findings in the current study, the results in the study have to be interpreted in the light of a number of limitations. Firstly, network analysis assumes that mental disorders (and therefore COVID anxiety) are causal systems. However, as we used cross-sectional data in the current study, causality cannot be securely assumed. At best, we were able to eliminate spurious 
candidates for causal relations. Causality assessment would require longitudinal data, collected repeatedly. Further studies may wish to examine such concerns, using longitudinal network analysis. Secondly, as we conducted the network analysis using a normative-community sample, the findings cannot be directly generalized to other samples, like specific racial and clinical groups. Thirdly, as we used a self-rating measure of COVID anxiety, the findings may not be applicable to data collected via clinical interviews, or from other sources. Fourthly, as our findings are based on group-level analyses, it may not be directly applicable at the individual level. It is possible that some of the associations found in the current study may not be applicable to some individuals. Clearly, we need more network studies of the COVID anxiety symptoms, using longitudinal data, collected using multiple sources and methods and different racial and clinical groups. Individualized networks would also be beneficial for a comprehensive understanding of the COVID anxiety network. Despite these limitations, our findings do offer novel insights on the structure of COVID anxiety symptoms, and their relative importance that can be used effectively for theorizing, assessing and treating COVID anxiety.

\section{Conclusions}

To conclude, the results of this study indicate that in terms of the importance or central 'strength' of each symptom, abdominal stress and tonic immobility are of the greatest centrality with dizziness being of the lowest. Furthermore, whilst causality cannot be concluded by the present study, edge weights meeting at least the moderate effect size cut-off were present for tonic immobility with dizziness, sleep disturbances and abdominal distress, and abdominal distress with appetite loss. This may indicate a potential causal link worthy of future investigation.

\section{Abbreviations}

CAS $=$ Covid Anxiety Scale

FCV $=$ Fear of COVID-19 Scale

CSS $=$ Covid Stress Scales

FCQ $=$ Fear of Coronavirus Scale

GAD = Generalized Anxiety Disorder

CFA = Confirmatory Factor Analysis

SEM $=$ Structural Equation Modelling

DASS $21=$ Depression Anxiety Stress Scale-21

WHO $=$ World Health Organisation 
AUDIT = Alcohol Use Disorders Identification Test

PLIS = Plain Language Information Statement

g-lasso $=$ Graphical Least Absolute Shrinkage and Selection Operator

JASP = Jeffrey's Amazing Statistics Program

CS $=$ Correlation Stability

\section{Declarations}

\section{Ethics approval and consent to participate}

All procedures performed in the study involving human participants were in accordance with the ethical standards of the institutional and/or national research committee and with the 1964 Helsinki declaration and its later amendments or comparable ethical standards. Informed consent was obtained from all individual participants included in the study. This article does not contain any studies with animals performed by any of the authors nor any studies making use of human tissue.

Thus, the present study was approved by the Human Ethics Research Committee of Victoria University (Australia).

\section{Consent for publication}

Not Applicable.

\section{Availability of data and materials}

Data is deposited as a supplementary file with the current document.

\section{Competing interests}

The authors of the present study declare they have no competing interests.

\section{Funding}

Dr Vasileios Stavropoulos received funding by:

- The Victoria University, Early Career Researcher Fund ECR 2020, number 68761601.

- The Australian Research Council, Discovery Early Career Researcher Award, 2021, number DE210101107

\section{Authors contributions}


RG: Contributed to the article's conceptualization, data curation, formal analysis, methodology, project administration, and writing of the original draft.

DTP: Reviewed and improved upon the final form of the manuscript. Compiled the manuscript and associated documents into submittable forms and corresponded with the publishing journal

SW: Contributed to the conceptualization of the study, reviewing and editing the final draft

VS: Contributed to the conceptualization of the study, reviewing and editing the final draft.

\section{Ackowledgements}

Not applicable

\section{Authors' information}

Dr Rapson Gomez: Rapson Gomez is adjunct professor in clinical psychology at Federation University, Australia. His research interests cover childhood and adolescent psychopathology, biological models of personality, cyberpsychology, and psychometrics.

Deon Tullett-Prado: Deon Tullett-Prado is a PhD candidate and emerging researcher in the area of behavioral addictions and in particular Internet Gaming Disorder. His expertise involves advanced statistical analysis skills and innovative techniques regarding population profiling.

Dr Watson is a senior lecturer in the School of Science, Psychology and Sport at Federation University.. With a research interest in shame and guilt and the ways in which these variables interact with personality, Dr Watson has made numerous research contributions in the fields of Clinical and Developmental Psychology.

Dr Vasileios Stavropoulos: Dr Vasileios Stavropoulos is a member of the Australian Psychological Society (APS) and a registered psychologist endorsed in Clinical Psychology with the Australian Health Practitioner Regulation Authority (AHPRA). Vasileios' research interests include the areas of Behavioral Addictions and Developmental Psychopathology. In that context, Vasileios is a member of the European Association of Developmental Psychology (EADP) and the EADP Early Researchers Union. Vasileios has received the ARC DECRA award 2021.

\section{References}

1. Ahorsu, D. K., Lin, C. Y., Imani, V., Saffari, M., Griffiths, M. D., \& Pakpour, A. H. (2020). The fear of COVID-19 scale: development and initial validation. International journal of mental health and addiction, 1-9.

2. Arora, A., Jha, A. K., Alat, P., \& Das, S. S. (2020). Understanding coronaphobia. Asian Journal of Psychiatry, 54, 102384. 
3. Babor, T. F., Hofmann, M., DelBoca, F. K., Hesselbrock, V., Meyer, R. E., Dolinsky, Z. S., \& Rounsaville, B. (1992). Types of alcoholics, I: Evidence for an empirically derived typology based on indicators of vulnerability and severity. Archives of general psychiatry, 49(8), 599-608.

4. Borgatti, S. P. (2005). Centrality and network flow. Social networks, 27, 55-71.

5. Borsboom, D., \& Cramer, A. O. (2013). Network analysis: an integrative approach to the structure of psychopathology. Annual Review of Clinical Psychology, 9, 91-121.

6. Boschloo, L., van Borkulo, C. D., Rhemtulla, M., Keyes, K. M., Borsboom, D., \& Schoevers, R. A. (2015). The network structure of symptoms of the diagnostic and statistical manual of mental disorders. Plos one, 10(9), e0137621.

7. Bringmann, L. F., Elmer, T., Epskamp, S., Krause, R. W., Schoch, D., Wichers, M. (2019). What do centrality measures measure in psychological networks? Journal of Abnormal Psychology. 128, 892-903.

8. Burkova, V. N., Butovskaya, M. L., Randall, A. K., Fedenok, J. N., Ahmadi, K., Alghraibeh, A. M., ... \& Zinurova, R. I. (2021). Predictors of anxiety in the COVID-19 pandemic from a global perspective: Data from 23 countries. Sustainability, 13(7), 4017.

9. CNU. The Coronavirus Anxiety Project. Retrieved from https://sites.google.com/cnu.edu/coronavirusanxiety.project/home).

10. Chen, J., Liu, X., Wang, D., Jin, Y., He, M., Ma, Y., ... \& Hou, X. (2021). Risk factors for depression and anxiety in healthcare workers deployed during the COVID-19 outbreak in China. Social psychiatry and psychiatric epidemiology, 56(1), 47-55.

11. Dawel, A., Shou, Y., Smithson, M., Cherbuin, N., Banfield, M., Calear, A. L., ... \& Batterham, P. J. (2020). The effect of COVID-19 on mental health and wellbeing in a representative sample of Australian adults. Frontiers in psychiatry, 11, 1026.

12. Epskamp S, Rhemtulla MT, Borsboom D. (2017). Generalized Network Psychometrics: Combining Network and Latent Variable Models. Psychometrika, 82, 904-927.

13. Epskamp, S., \& Fried, E. I. (2018). A tutorial on regularized partial correlation networks. Psychological Methods, 23, 617-634.

14. Epskamp, S., Borsboom, D., \& Fried, E. I. (2018). Estimating psychological networks and their accuracy: A tutorial paper. Behavior Research Methods, 50, 195-212.

15. Epskamp, S., Cramer, A. O., Waldorp, L. J., Schmittmann, V. D., \& Borsboom, D. (2012). qgraph: Network visualizations of relationships in psychometric data. Journal of Statistical Software, 48, 118.

16. Evren, C., Evren, B., Dalbudak, E., Topcu, M., \& Kutlu, N. (2020). Measuring anxiety related to COVID19: A Turkish validation study of the Coronavirus Anxiety Scale. Death Studies, 1-7.

17. Ferguson, C. J. (2009). An effect size primer: A guide for clinicians and researchers. Professional Psychology Research and Practice, 40, 532-538. 
18. Foygel, R., \& Drton, M. (2010). Extended Bayesian information criteria for Gaussian graphical models. arXiv preprint arXiv.1011.6640.

19. Fried, E. I., Bockting, C., Arjadi, R., Borsboom, D., Amshoff, M., Cramer, A. O. J., Epskamp, S., Tuerlinckx, F., Carr, D., \& Stroebe, M. (2015). From loss to loneliness: The relationship between bereavement and depressive symptoms. Journal of Abnormal Psychology, 124, 256-265.

20. Fruchterman, T. M., \& Reingold, E. M. (1991). Graph drawing by force-directed placement. Software: Practice and Experience, 21, 1129-1164.

21. Galea S., Merchant, R. M., Lurie, N. (2020). The mental health consequences of COVID-19 and physical distancing: the need for prevention and early intervention. JAMA Intern Med., 180, 817-818.

22. Gasteiger, N., Vedhara, K., Massey, A., Jia, R., Ayling, K., Chalder, T., ... \& Broadbent, E. (2021). Depression, anxiety and stress during the COVID-19 pandemic: results from a New Zealand cohort study on mental well-being. BMJ open, 11(5), e045325.

23. Gomez, R. (2013). Depression Anxiety Stress Scales: Factor structure and differential item functioning across women and men. Personality and Individual Differences, 54(6), 687-691.

24. Heeren, A., Bernstein, E. E., \& McNally, R. J. (2018). Deconstructing trait anxiety: A network perspective. Anxiety, Stress, \& Coping, 31, 262-276.

25. Huang, Y., \& Zhao, N. (2020). Generalized anxiety disorder, depressive symptoms and sleep quality during COVID-19 outbreak in China: a web-based cross-sectional survey. Psychiatry research, 288, 112954.

26. Isvoranu, A. M., Boyette, L. L., Guloksuz, S., \& Borsboom, D. (2017). Chapter G1. Symptom network models of psychosis. Dimensions of Psychosis. Oxford University Press.

27. Krämer, N., Schäfer, J., \& Boulesteix, A. L. (2009). Regularized estimation of large-scale gene association networks using graphical Gaussian models. BMC Bioinformatics, 10, 1-24.

28. Lee, D. (2019). The convergent, discriminant, and nomological validity of the Depression Anxiety Stress Scales-21 (DASS-21). Journal of affective disorders, 259, 136-142.

29. Lee, S. A. (2020a). Coronavirus anxiety scale: A brief mental health screener for covid-19 related anxiety. Death Studies. https://doi.org/10.1080/07481187.2020.1748481

30. Lee, S. A. (2020b). Measuring coronaphobia: the psychological basis of the Coronavirus Anxiety Scale. Dusunen Adam, 33(2), 107-108.

31. Lee, S. A., Mathis, A. A., Jobe, M. C., \& Pappalardo, E. A. (2020). Clinically significant fear and anxiety of COVID-19: A psychometric examination of the Coronavirus Anxiety Scale. Psychiatry Research, $290,113112$.

32. Lovibond, P. F., \& Lovibond, S. H. (1995). The structure of negative emotional states: Comparison of the Depression Anxiety Stress Scales (DASS) with the Beck Depression and Anxiety Inventories. Behaviour research and therapy, 33(3), 335-343.

33. Mertens, G., Gerritsen, L., Duijndam, S., Salemink, E., \& Engelhard, I. M. (2020). Fear of the coronavirus (COVID-19): Predictors in an online study conducted in March 2020. Journal of anxiety disorders, 74, 
102258.

34. McKay, D., Yang, H., Elhai, J., Asmundson, G. J. G. (2020). Anxiety regarding contracting COVID-19 related to interoceptive anxiety sensations: The moderating role of disgust propensity and sensitivity. Journal of Anxiety Disorders, 73:102233

35. McNally, R. J., Heeren, A., \& Robinaugh, D. J. (2017). A Bayesian network analysis of posttraumatic stress disorder symptoms in adults reporting childhood sexual abuse. European Journal of Psychotraumatology, 8 (sup3), 1341276.

36. Mullarkey, M. C., Marchetti, I., \& Beevers, C. G. (2019). Using network analysis to identify central symptoms of adolescent depression. Journal of Clinical Child \& Adolescent Psychology, 48, 656-668.

37. Nikčević, A. V., \& Spada, M. M. (2020). The COVID-19 anxiety syndrome scale: Development and psychometric properties. Psychiatry research, 292, 113322.

38. Norton, P. J. (2007). Depression Anxiety and Stress Scales (DASS-21): Psychometric analysis across four racial groups. Anxiety, stress, and coping, 20(3), 253-265.

39. Opsahl, T., Agneessens, F., \& Skvoretz, J. (2010). Node centrality in weighted networks: Generalizing degree and shortest paths. Social Networks, 32, 245-251.

40. Pfefferbaum, B., \& North, C. S. (2020). Mental health and the Covid-19 pandemic. New England Journal of Medicine, 383(6), 510-512.

41. Ramos-Vera, C, A. (2021). The internal dynamics of the Coronavirus Anxiety Scale through network analysis. Revista Colombiana de Psiquiatría. On line first. doi: https://doi.org/10.1016/j.rcp.2020.12.008

42. Salari, N., Hosseinian-Far, A., Jalali, R., Vaisi-Raygani, A., Rasoulpoor, S., Mohammadi, M., ... \& Khaledi-Paveh, B. (2020). Prevalence of stress, anxiety, depression among the general population during the COVID-19 pandemic: a systematic review and meta-analysis. Globalization and health, 16(1), 1-11.

43. Santos Jr, H. P., Kossakowski, J. J., Schwartz, T. A., Beeber, L., \& Fried, E. I. (2018). Longitudinal network structure of depression symptoms and self-efficacy in low-income mothers. Plos One, 13(1), e0191675.

44. Saunders, J. B., Aasland, O. G., Babor, T. F., De la Fuente, J. R., \& Grant, M. (1993). Development of the alcohol use disorders identification test (AUDIT): WHO collaborative project on early detection of persons with harmful alcohol consumption- II. Addiction, 88(6), 791-804.

45. Shah, S. M. A., Mohammad, D., Qureshi, M. F. H., Abbas, M. Z., \& Aleem, S. (2021). Prevalence, Psychological Responses and associated correlates of depression, anxiety and stress in a global population, during the coronavirus disease (COVID-19) pandemic. Community mental health journal, 57(1), 101-110.

46. Skalski, S., Uram, P., Dobrakowski, P., \& Kwiatkowska, A. (2021). The link between ego- resiliency, social support, SARS-CoV-2 anxiety and trauma effects. Polish adaptation of the Coronavirus Anxiety Scale. Personality and individual differences, 171, 110540. 
47. Stanton, R., To, Q. G., Khalesi, S., Williams, S. L., Alley, S. J., Thwaite, T. L., ... \& Vandelanotte, C. (2020). Depression, anxiety and stress during COVID-19: associations with changes in physical activity, sleep, tobacco and alcohol use in Australian adults. International journal of environmental research and public health, 17(11), 4065.

48. Taylor, S., Landry, C. A., Paluszek, M. M., Fergus, T. A., McKay, D., \& Asmundson, G. J. (2020). Development and initial validation of the COVID Stress Scales. Journal of Anxiety Disorders, 72, 102232.

49. Team, JASP. (2018). JASP (Version 0.9). Retrieved from https://jasp-stats.org.

50. Tibshirani, R. (1996). Regression shrinkage and selection via the lasso. Journal of the Royal Statistical Society: Series B (Methodological), 58, 267-288.

51. Xiong, J., Lipsitz, O., Nasri, F., Lui, L. M., Gill, H., Phan, L., ... \& Mclntyre, R. S. (2020). Impact of COVID19 pandemic on mental health in the general population: A systematic review. Journal of affective disorders.

52. Yang, Z., Algesheimer, R., \& Tessone, C. J. (2016). A comparative analysis of community detection algorithms on artificial networks. Scientific Reports, 6, 30750.

\section{Tables}

Table 1 Descriptives, and Weights Matrix Between the CAS COVID Anxiety Symptoms from the Network Analysis

\begin{tabular}{llllll} 
Variable \#/Brief description & 1 & 2 & 3 & 4 & 5 \\
\hline 1 Dizziness & 0.00 & 0.14 & 0.33 & 0.03 & 0.15 \\
\hline 2 Sleep Disturbances & & 0.00 & 0.30 & 0.17 & 0.16 \\
\hline 3 Tonic Immobility & & 0.00 & 0.09 & 0.35 \\
\hline 4 Appetite Loss & & & 0.00 & 0.52 \\
\hline 5 Abdominal Distress & & & & & 0.00 \\
\hline Mean (Standard Deviation) & $.23(.58)$ & $.48(.78)$ & $.29(.67)$ & $.26(.66)$ & $.30(.69)$
\end{tabular}

Table 2 Centrality Indices of CAS COVID Anxiety Symptoms from the Network Analysis 


\begin{tabular}{lllll} 
Variable \#/Brief description & Betweenness & Closeness & Strength & Expected Influence \\
\hline 1 Dizziness & -0.67 & -0.98 & -1.12 & -1.12 \\
\hline 2 Sleep Disturbances & -0.67 & -0.60 & -0.57 & -0.57 \\
\hline 3 Tonic Immobility & $\underline{1.57}$ & $\underline{1.55}$ & $\underline{0.76}$ & $\underline{0.76}$ \\
\hline 4 Appetite Loss & -0.67 & -0.36 & -0.37 & -0.37 \\
\hline 5 Abdominal Distress & $\underline{0.45}$ & $\underline{0.39}$ & $\underline{1.30}$ & $\underline{1.30}$
\end{tabular}

Note. Higher numbers indicate that the variable is more central to the network; highest two values are underlined within each index.

Table 3 Weights Matrix for the CAS Symptoms with Distress and Alcohol Use from the Expanded Network Analysis

\begin{tabular}{|lll|}
\hline CAS Symptoms & Distress & Alcohol use \\
\hline Dizziness & 0.11 & 0.06 \\
\hline Sleep Disturbances & 0.00 & 0.00 \\
\hline Tonic Immobility & 0.13 & -0.03 \\
\hline Appetite Loss & 0.13 & -0.03 \\
\hline Abdominal Distress & 0.00 & 0.08 \\
\hline Mean (Standard Deviation) & $20.12(13.80)$ & $4.47(6.00)$ \\
\hline
\end{tabular}

\section{Figures}




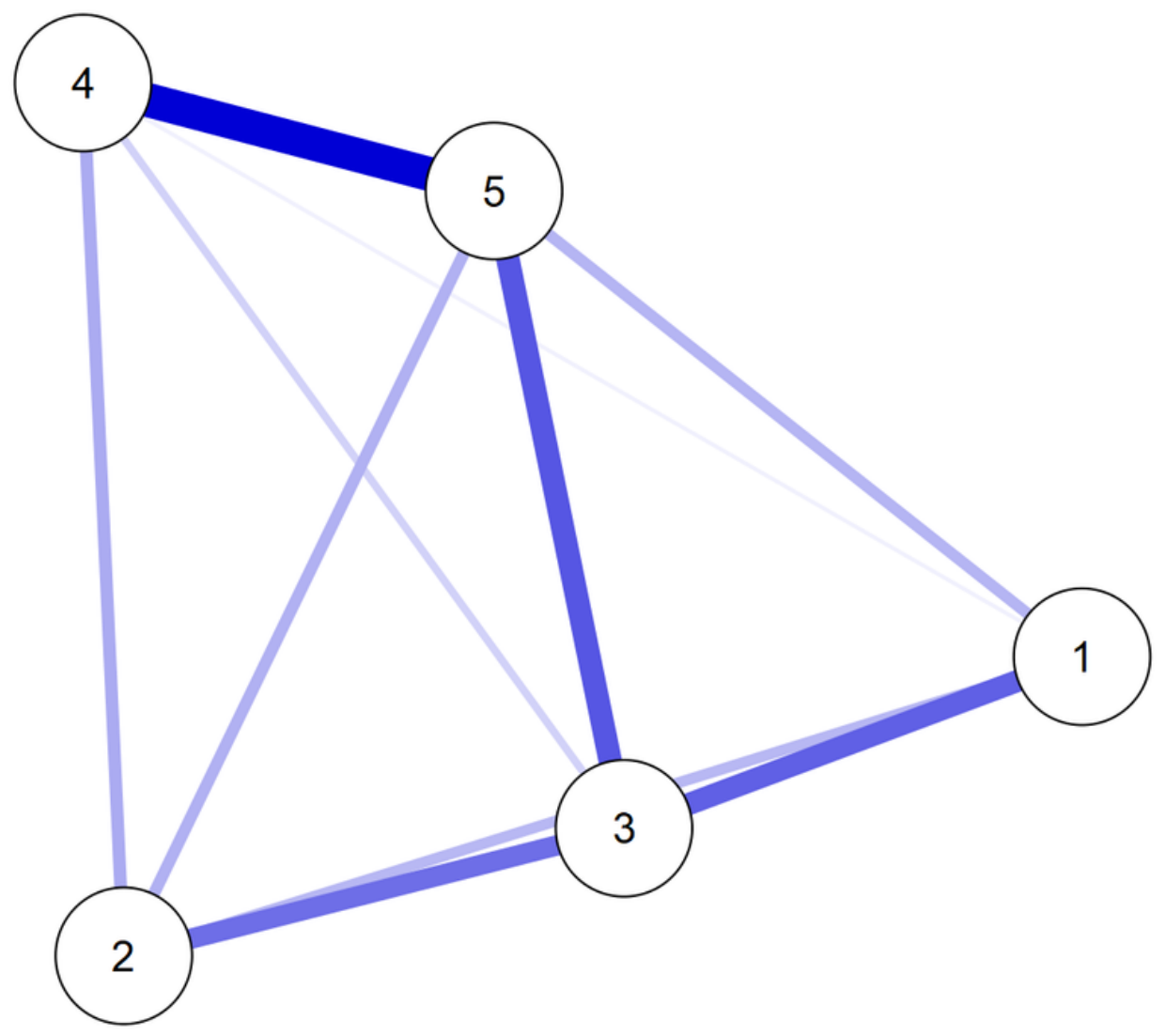

1: Dizziness

2: SleepDisturbances

3: Toniclmmobility

4: AppetiteLoss

5: AbdominalDistress

Figure 1

Network of the CAS COVID Anxiety Symptoms 

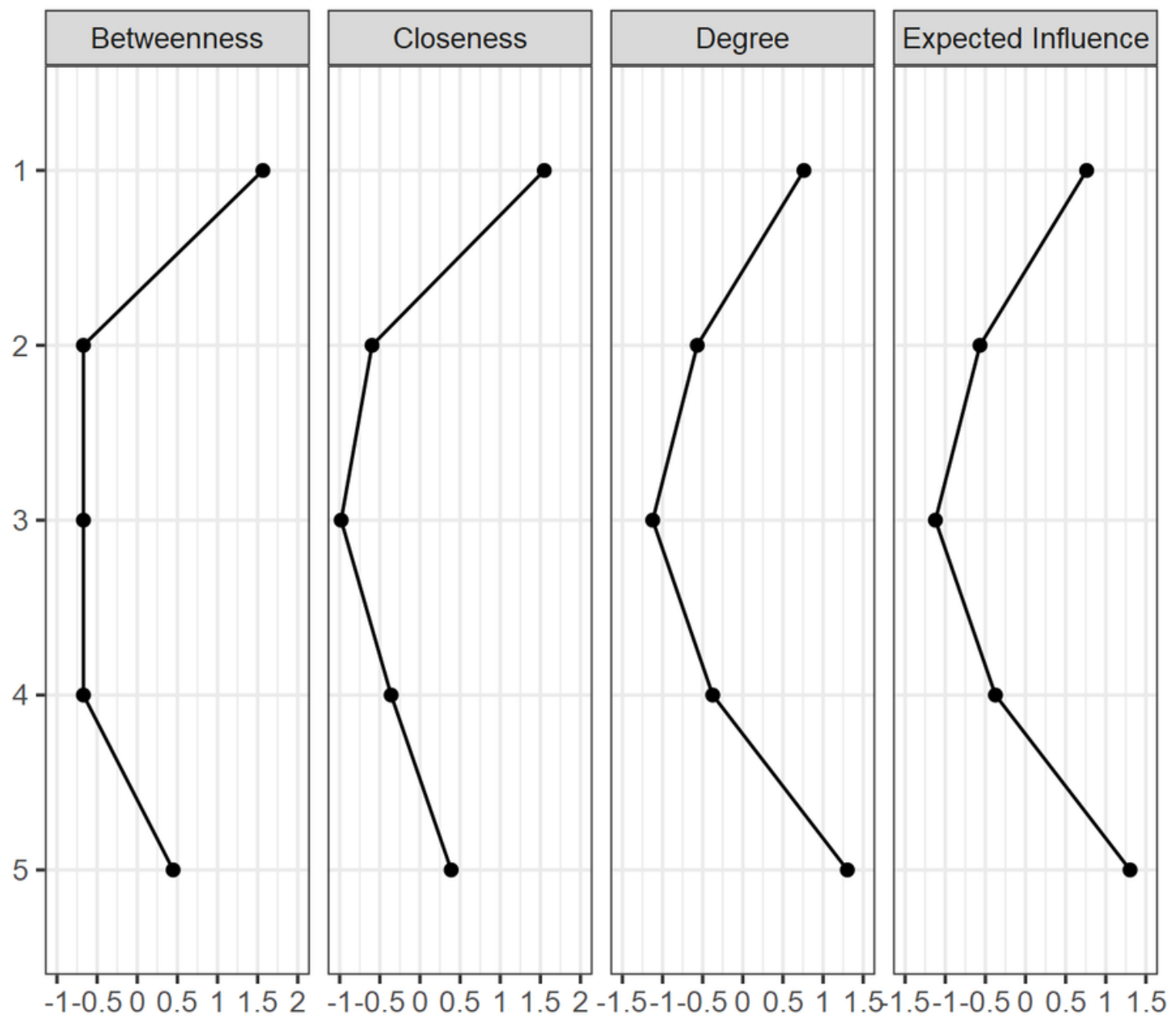
1 = TonicImmobility
2 = SleepDisturbances
$3=$ Dizziness
$4=$ AppetiteLoss
$5=$ AbdominalDistress

\section{Figure 2}

Centrality Plots (Betweenness, Closeness, Degree, and Expected Influence) in the Network for the Association in the Network of Each Node of the CAS COVID Anxiety Symptoms

\section{Supplementary Files}

This is a list of supplementary files associated with this preprint. Click to download.

- SupplementaryFigureLegends.docx

- SupplementaryFigureS1EdgeStabilityEstimatesforCAS.tif

- SupplementaryFigureS2StabilityofCASCentrallndices.tif 